\title{
Diffractive W production in SCI models
}

\author{
Dominik Werder ${ }^{* a}$, Gunnar Ingelman ${ }^{a}$, Roman Pasechnik ${ }^{b}$, Johan Rathsman ${ }^{b}$ \\ ${ }^{a}$ Uppsala University, Box 516, SE-75120 Uppsala, Sweden \\ ${ }^{b}$ Astronomy and Theoretical Physics, Lund University, Sölvegatan 14A, SE-22362 Lund, Sweden \\ E-mail: Dominik. Werder@physics.uu. se Gunnar. Ingelman@physics.uu. se, \\ Roman.Pasechnik@thep.lu.se, Johan.Rathsman@thep.lu.se,
}

\begin{abstract}
The soft color interaction model (SCI) as an add-on to standard perturbative QCD describes diffractive processes by allowing for additional soft color-octet exchanges below the conventional threshold for parton showering. We discuss how diffractive and inclusive event samples for diffractive hard processes can be obtained from a Monte Carlo in a unified way for $p p \rightarrow p\left[W^{ \pm}+X\right] p$ as an example, and discuss potential differences compared with Regge based models. In particular we address the question of a possible $W^{ \pm}$charge asymmetry and show that this observable approaches the result from pomeron based models in the limit of very forward final state protons.
\end{abstract}

The European Physical Society Conference on High Energy Physics - EPS-HEP2013

18-24 July 2013

Stockholm, Sweden

\footnotetext{
*Speaker.
} 


\section{Soft color exchange models}

Despite considerable progress in recent years, diffractive processes remain a challenge for the theoretical description. In the case of diffractive hard processes there exists a partonic subprocess with a hard scale which allows a perturbative calculation. The soft QCD effects which give rise to the diffractive signatures like large rapidity gaps (LRG) or leading beam particles, are much less well understood. Different approaches exist to describe the soft effects in such observables. Regge theory invokes the hadron-like color-neutral pomeron [1] while soft color interaction models on the other hand are based on the exchange of color charge between the hard subprocess and the beam remnant, first introduced as SCI in [2] and later evolved as the generalized area law GAL in [3]. Momenta in the event stay almost unchanged by such exchanges whereas the color topology within the event can change, resulting in an effective overall color-singlet, electrically neutral $t$ channel exchange and observable effects during hadronization. One important difference between soft color exchange and pomeron based models is that a sample based on a cut on the forward beam particle momentum fraction $z$ can contain events where the $t$-channel exchange between the hard subprocess and the beam is not effectively color-singlet because final state beam particles of sizeable momentum fraction can also be obtained from regular diquark fragmentation. Recently diffractive gauge boson production was considered as a sensitive way to investigate the production mechanism [4] based on a measurement of $A=\left(W^{+}-W^{-}\right) /\left(W^{+}+W^{-}\right)$, explored in the context of soft color exchange models in [5].

\section{2. $W^{ \pm}$asymmetry as a test of the underlying mechanism}

Figs. 2, 3 and 4 show the results for the process illustrated in Fig. 1. Several model parameters have a large influence on the spectrum of leading particles, e.g. the quark masses which influence the available phase space for hadronization into leading protons, the choice whether one allows radiation from the remnant, and the model of multiple interactions. To illustrate these dependen-
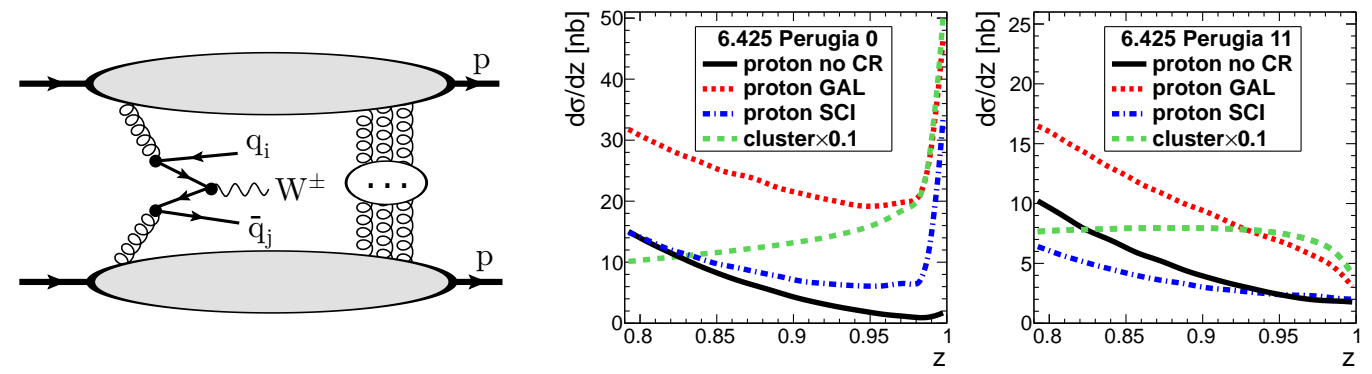

Figure 1: (left) The exclusive diffractive process $p p \rightarrow p\left[W^{ \pm} X\right] p$ with central $W^{ \pm}+2$ jets as the hard subprocess, separated from the final protons, based on soft color exchanges in QCD.

Figure 2: (right) Distribution in momentum fraction $z=\left|p_{z}\right| / p_{\text {beam }}$ of the single leading proton in $p p \rightarrow p\left[W^{ \pm} X\right] p$ events at $\sqrt{s}=14 \mathrm{TeV}$ obtained from Pythia [6], without and with soft exchanges [7], for two sets of tuned Monte Carlo parameters (Perugia 0 and 11 [8]) which affect among others the remnant treatment. Leading clusters with $m_{\mathrm{cl}}<1.5 \mathrm{GeV}$ and proton flavor quantum numbers, but not necessarily colour singlets, are scaled down to overlap with the diffractive proton peak at $z \rightarrow 1$. 


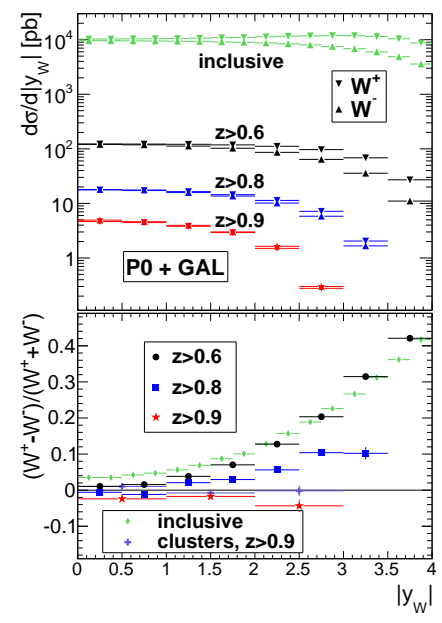

Figure 3: (left) Cross sections in rapidity $y_{W}$ and corresponding charge asymmetries for the GAL model. Charge asymmetry is strongly dependent on the chosen cut $z$.

Figure 4: (right) Cross sections and asymmetry without color reconnections (CR) and with the soft color exchange models SCI and GAL, depending on the cut $z$ and requiring a similar forward momentum fraction on both sides.

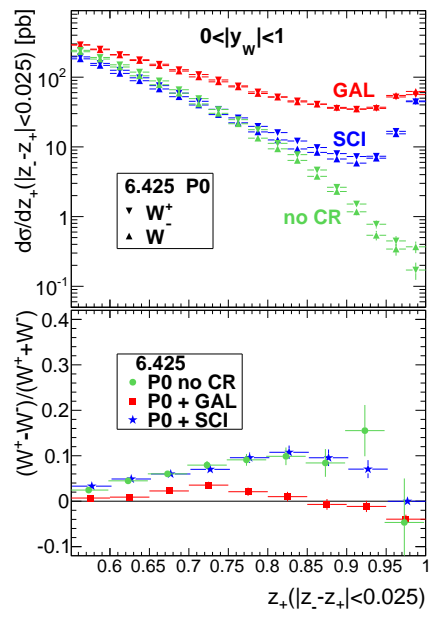

cies, Fig. 2 shows the single-sided cross section of forward protons and proton-like 'clusters' for different monte carlo models, where clusters are defined as combinations of partons with total proton flavor, $|m|<1.5 \mathrm{GeV}$, but not necessarily color-singlet. The Monte Carlo tune Perugia 11 [8] in Fig. 2 treats also the constituents of the remnant as resolvable partons and allows the dipoles stretched between the central system and the remnant to radiate, which eliminates the forward peak in the spectrum of final state protons. The spectrum is also sensitive to the modelling of multiple interactions (MI) because older approaches treat the MI seperately from showering, which effectively allows secondary interactions above the scale chosen as the factorization scale of the primary interaction. Newer models intertwine the MI and the showers by using a common Sudakov factor. Fig. 2 also illustrates how the leading proton spectrum due to regular diquark fragmentation blends into the forward diffractive peak region, which illustrates the importance of a careful choice of the cut on $z$ when comparing soft color exchange and Regge based models. Especially the $W$ charge asymmetry goes to zero when the cut $z$ approaches the forward limit as shown in Figs.3 and 4.

\section{References}

[1] J. R. Forshaw and D. A. Ross, Quantum chromodynamics and the pomeron, Cambridge Lect. Notes Phys. 9, 1 (1997).

[2] A. Edin, G. Ingelman and J. Rathsman, Soft color interactions as the origin of rapidity gaps in DIS, Phys. Lett. B 366, 371 (1996); Z.Phys. C75, 57 (1997).

[3] J. Rathsman, A generalised area law for hadronic string reinteractions, Phys. Lett. B 452 (1999) 364.

[4] K. Golec-Biernat, C. Royon, L. Schoeffel and R. Staszewski, Electroweak vector boson production at the LHC as a probe of mechanisms of diffraction, Phys. Rev. D 84, 114006 (2011).

[5] G. Ingelman, R. Pasechnik, J. Rathsman and D. Werder, Diffractive W production at hadron colliders as a test of colour singlet exchange mechanisms, Phys. Rev. D 87, 094017 (2013) .

[6] T. Sjöstrand, S. Mrenna and P. Z. Skands, PYTHIA 6.4 Physics and Manual, JHEP 0605, 026 (2006)

[7] D. Eriksson, J. Rathsman, O. Stål, Implementation of SCI and GAL colour reconnections models in Pythia 6.4, unpublished, available on http://home.thep.lu.se/ rathsman/scigal

[8] P. Z. Skands, Tuning Monte Carlo Generators: The Perugia Tunes, Phys. Rev. D 82, 074018 (2010). 